\title{
Band gaps and the electromechanical coupling coefficient of a surface acoustic wave in a two-dimensional piezoelectric phononic crystal
}

\author{
Tsung-Tsong Wu, ${ }^{*}$ Zin-Chen Hsu, and Zi-Gui Huang \\ Institute of Applied Mechanics, National Taiwan University, Taipei 106, Taiwan \\ (Received 26 April 2004; revised manuscript received 6 October 2004; published 28 February 2005)
}

\begin{abstract}
In this paper we analyze the phononic band structure of bulk and surface waves in a two-dimensional periodic structure consisting of an array of piezoelectric cylinders in an isotropic background material. The explicit formulations of the bulk wave and the surface wave dispersion relations in such a structure are derived based on the plane wave expansion method. The band gaps and the electromechanical coupling coefficients for surface acoustic waves propagating in such a structure are calculated. The influences of material and geometrical anisotropy as well as the filling fraction on the electromechanical coupling coefficients are discussed, and some silent features are drawn. The results of this research may serve as an important reference for studying surface acoustic wave devices consisting of phononic structures.
\end{abstract}

DOI: 10.1103/PhysRevB.71.064303 PACS number(s): 63.20.-e, 43.20.+g, 43.40. + s, 46.40.Cd

Interest in the propagation of acoustic waves in phononic crystals has grown in recent years because of the analogy with photonic crystals. The existence of elastic band gaps in phononic crystals may lead to the development of a variety of acoustic devices, such as filters and transducers. In the past, several theories of acoustic band structures have been developed. Kushwaha et al. ${ }^{1}$ utilized the plane wave expansion method to calculate the first full band structure of the transverse polarization mode for periodic elastic composites. In addition, Wilm et $a .^{2}$ used the same method to study the out-of-plane propagation of elastic bulk waves in a twodimensional phononic crystal, which consists of array of parallel square-section piezoelectric rods of Z-cut quartz embedded in an epoxy matrix. In Refs. 3-6, the multiple scattering theory was applied to study the band gaps of threedimensional periodic acoustic composites and the band structure of a phononic crystal consisting of complex and frequency-dependent Lamè coefficients. García-Pablos et al. ${ }^{7}$ used the finite difference time domain method to interpret the experimental data of two-dimensional systems consisting of cylinders of fluids ( $\mathrm{Hg}$, air, and oil) inserted periodically in a finite slab of $\mathrm{Al}$ host.

The investigation of surface acoustic waves propagating in such composites has also receiving increasing attention. It is certainly more complicated to study the surface acoustic waves than that of the bulk acoustic waves due to the additional traction free boundary conditions. Tanaka and Tamura $^{8,9}$ reported calculations for surface waves on a square lattice consisting of cubic materials (AlAs/GaAs) and on a hexagonal lattice consisting of isotropic materials ( $\mathrm{Al} /$ polymer) using the plane wave expansion method. Tartakovskaya ${ }^{10}$ employed a procedure similar to the socalled empty lattice of solid state physics to investigate the existence of full band gap of surface waves in elastic periodic composites. Wu et al. ${ }^{11}$ utilized the plane wave expansion method to study the phononic band gaps of surface waves in two-dimensional phononic structures consisting of general anisotropic materials. Wilm et al. ${ }^{12}$ applied the plane wave expansion method to address piezoelectric composite plate structures and analyses the dispersion relations of plate modes and related characteristics. Among the existing literature, we note that the study of the behavior of surface waves propagating in periodic piezoelectric structures is still lacking.

On taking the piezoelectricity and electrical excitation conditions into account, this paper is to investigate theoretically the characteristics of the bulk waves and surface acoustic waves in two-dimensional periodic piezoelectric structures based on the plane wave expansion method. The influence of the piezoelectricity on the band gap characteristics and wave propagating properties are calculated and discussed.

Consider a phononic crystal composed of a two dimensional periodic array ( $x-y$ plane) of material $A$ embedded in a background material $B$. Both $A$ and $B$ materials are piezoelectric or dielectric. In the quasistatic approximation, the governing field equations of piezoelectricity can be expressed as

$$
\begin{gathered}
\rho \ddot{u}_{j}=\partial_{i} T_{i j}, \\
\partial_{i} D_{i}=0,
\end{gathered}
$$

where $\rho(\mathbf{x})$ is the position-dependent mass density and $u_{j}(\mathbf{r}, t)$ is the displacement vector, $\mathbf{r}=(\mathbf{x}, z)=(x, y, z)$ is the position vector, and $T_{i j}(\mathbf{r}, t)$ and $D_{i}(\mathbf{r}, t)$ are the stress and electric displacement, respectively. The piezoelectric constitutive relations with the displacement $u_{j}(\mathbf{r}, t)$ and the electric potential $\phi(\mathbf{r}, t)$ as variables are given by

$$
\begin{gathered}
T_{i j}=c_{i j k l} \partial_{l} u_{k}+e_{l i j} \partial_{l} \phi, \\
D_{i}=e_{i k l} \partial_{l} u_{k}-\varepsilon_{i l} \partial_{l} \phi,
\end{gathered}
$$

where $c_{i j k l}(\mathbf{x}), e_{l i j}(\mathbf{x})$, and $\varepsilon_{i l}(\mathbf{x})$ are the position-dependent elastic stiffness constants, piezoelectric constants, and permittivity, respectively. Note that the material constants do not dependent on the coordinate $z$ because of the homogeneity of the phononic crystal along the $z$ axis. Due to the spatial periodicity, the material constants can be expanded in Fourier 
Dispersion Relations, Cylinder: $\mathrm{Bi}_{12} \mathrm{GeO}_{20} / \mathrm{Base}: \mathrm{SiO}_{2} / f=0.6$, sq.

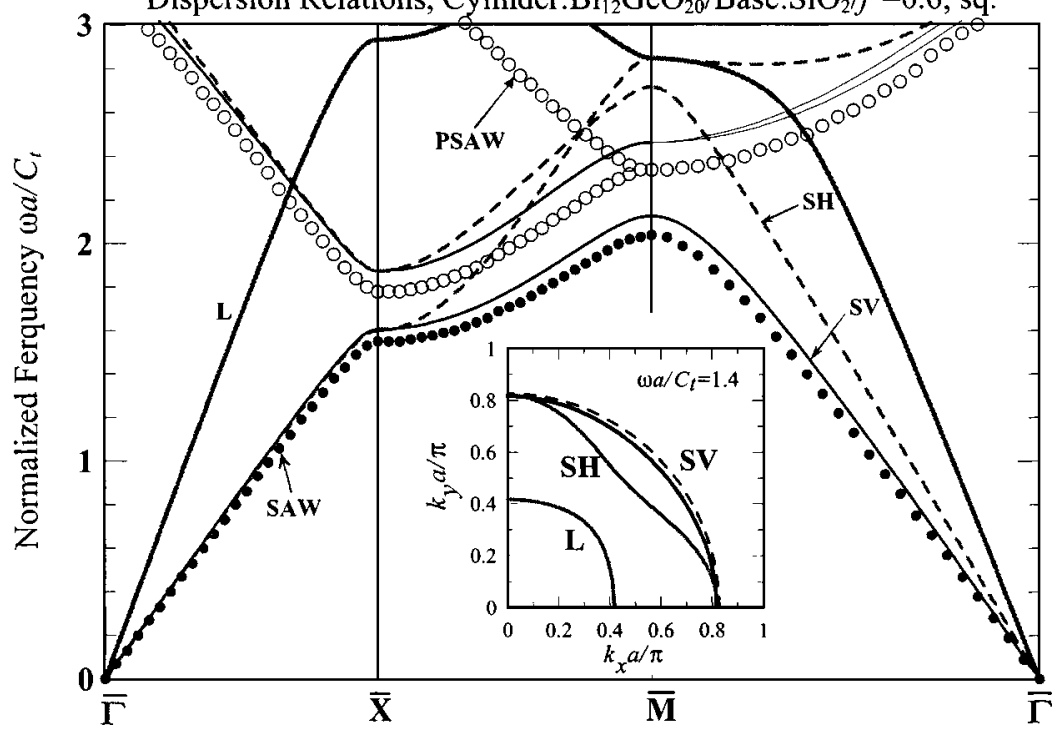

FIG. 1. Dispersion relations of surface acoustic waves (SAW, dots), pseudo-SAW (PSAW, open circles), bulk longitudinal ( $L$, bold solid lines), shear vertical (SV, thin solid lines), and shear horizontal ( $\mathrm{SH}$, dashed lines) acoustic waves in a square lattice of $\mathrm{Bi}_{12} \mathrm{GeO}_{20}$ in $\mathrm{SiO}_{2}$. The inset shows the slowness surface (in $k_{x}-k_{y}$ plane) at $\omega a / C_{t}=1.4$ of the bulk waves. Dashed lines are calculated by ignoring the piezoelectricity (where $C_{t}=\sqrt{c_{44}^{B} / \rho^{B}}$ ). series with respect to the two-dimensional reciprocal lattice vectors $(\mathrm{RLVs}), \mathbf{G}=\left(G_{1}, G_{2}\right)$, as

$$
\alpha(\mathbf{x})=\sum_{\mathbf{G}} e^{i \mathbf{G} \cdot \mathbf{x}} \alpha_{\mathbf{G}},
$$

where $\alpha=\left(\rho, c_{i j k l}, e_{l i j}, \varepsilon_{i l}\right)$, and $\alpha_{\mathbf{G}}$ is the corresponding Fourier coefficients. On utilizing the Bloch theorem and expanding the displacement vector $u_{j}(\mathbf{r}, t)$ and electric potential $\phi(\mathbf{r}, t)$ in Fourier series, we have

$$
\begin{aligned}
& u_{j}(\mathbf{r}, t)=\sum_{\mathbf{G}} e^{i(\mathbf{k} \cdot \mathbf{x}-\omega t)}\left(e^{i \mathbf{G} \cdot \mathbf{x}} A_{\mathbf{G}}^{j} e^{i k_{z} z}\right), \\
& \phi(\mathbf{r}, t)=\sum_{\mathbf{G}} e^{i(\mathbf{k} \cdot \mathbf{x}-\omega t)}\left(e^{i \mathbf{G} \cdot \mathbf{x}} A_{\mathbf{G}}^{4} e^{i k_{z} z}\right),
\end{aligned}
$$

where $\mathbf{k}=\left(k_{1}, k_{2}\right)$ is the Bloch wave vector, $\omega$ is the circular frequency, and $k_{z}$ is the wave number along the $z$ direction, and $A_{\mathbf{G}}^{j},(j=1,2,3)$ and $A_{\mathbf{G}}^{4}$ are the amplitudes of the displacement vector and electric potential, respectively. Using Eqs. (3) and (4), the following generalized eigenvalue problem with respect to $k_{z}$ can be derived from Eq. (1) and Eq. (2): ${ }^{11,13}$

$$
\left(\mathbf{R} k_{z}^{2}+\mathbf{Q} k_{z}+\mathbf{P}\right) \mathbf{U}=0,
$$

where $\mathbf{U}=\left\{\mathbf{A}_{\mathbf{G}^{\prime}}^{1}, \mathbf{A}_{\mathbf{G}^{\prime}}^{2}, \mathbf{A}_{\mathbf{G}^{\prime}}^{3}, \mathbf{A}_{\mathbf{G}^{\prime}}^{4}\right\}^{T}$ is called the generalized displacement vector, the $4 n \times 4 n$ matrices $\mathbf{R}, \mathbf{Q}$, and $\mathbf{P}$ are functions of $\mathbf{k}, \mathbf{G}, \omega$, and the Fourier coefficients of material constants $\rho_{\mathbf{G}}, c_{\mathbf{G}}^{i j k l}, e_{\mathbf{G}}^{l i j}$ and $\varepsilon_{\mathbf{G}}^{i l}$, and $n$ is the number of RLVs. The expressions of the components of matrices addition to the case of phononic crystals with piezoelectricity ignored are listed in the Appendix.

The dispersion relations of bulk waves can be obtained by setting the determinant of matrix $\mathbf{P}$ equals to zero. But for the case of surface wave, the lattice displacement and the electric potential exponentially decay into the half space, and thus, the complex wave number $k_{z}$ with positive imaginary part, which obtained from Eq. (5), are chosen. Accordingly, we put $A_{\mathbf{G}}^{j(l)}=X_{l} a_{\mathbf{G}}^{j(l)}(j=1-4$ and $l=1-4 \mathrm{n})$, in Eq. (4) and sum over $l . a_{\mathbf{G}}^{j(l)}$ is the associated eigenvector of the eigenvalue $k_{z}^{(l)}$, and $X_{l}$ is the undetermined weighting coefficient which can be determined from the boundary conditions on the surface $z=0$. The mechanical boundary conditions at the free surface require the nullity of stress components on the surface, i.e., $\left.T_{i 3}\right|_{z=0}=\left.\left(c_{i 3 k l} \partial_{l} u_{k}+e_{l i 3} \partial_{l} \phi\right)\right|_{z=0}=0, \quad(i=1,2,3)$. For piezoelectric materials, the electric boundary condition should be specified. If the surface is covered by a hypothetical medium with zero permittivity, we have the open-circuit condition with $\left.D_{3}\right|_{z=0}=\left.\left(c_{i 3 k l} \partial_{l} u_{k}-\varepsilon_{3 l} \partial_{l} \phi\right)\right|_{z=0}$, giving the phase velocity $V=V_{0}$. If the surface is covered by a very thin metallic film, we have the short-circuit condition with $\left.\phi\right|_{z=0}=0$, giving the phase velocity $V=V_{\infty}$. By using the Ingebrigtsen approximation, ${ }^{14}$ the electromechanical coupling coefficient of a piezoelectric medium can be approximated as

$$
K^{2}=2 \frac{V_{0}-V_{\infty}}{V_{0}} .
$$

Apply the boundary conditions, we have a homogeneous linear system of equations for $X_{l}$, or $\mathbf{H} \cdot \mathbf{X}=0$. $\mathbf{H}$ is a $4 n \times 4 n$ matrix, and the explicit expressions for the components of $\mathbf{H}$ are given in the Appendix. For the existence of a nontrivial solution of $X_{l}$, the following condition must be satisfied,

$$
\operatorname{det}(\mathbf{H})=0 \text {. }
$$

Equations (5) and (7) can be solved simultaneously to determine the dispersion relation for surface waves propagating in the two-dimensional phononic crystals with both the filling material and the background material belong to the triclinic system.

We consider a two-dimensional phononic crystal with square lattice where the circular cylinders of radius $r_{0}$ are embedded periodically in background material with lattice spacing $a$. The reciprocal-lattice vector in these structures is $\mathbf{G}=\left(2 \pi N_{1} \pi / a, 2 \pi N_{2} / a\right)$ with integers $N_{1}$ and $N_{2}$.

Figure 1 shows the dispersion relations of piezoelectric waves corresponding to a two-dimensional square lattice 
phononic crystal consisting of $\mathrm{Bi}_{12} \mathrm{GeO}_{20}(001)$ circular cylinders in a silica substrate along the boundary of the irreducible part of the Brillouin zone. The filling fraction $f$ is 0.6. The notation (001) means the crystal plane parallel to the $x$ $-y$ plane. Material constants used in the calculation can be found in Ref. 15. In Fig. 1, we find that all bulk modes as well as the surface mode exhibit folding effect with frequency zgaps at the zone-edge $\bar{X}$ point, and the gaps of SH mode and surface/pseudosurface mode continuous to exist along the boundary up to the $M$ point. In order to analyze the influence of piezoelectric effect on the phononic band structure, we first calculated the slowness surface at $\omega a / C_{t}=1.4$, and the results are shown in the inset of Fig. 1. Only the shear vertical wave is piezoelectric, as shown by the gap between its curve and the dashed curve (obtained with piezoelectricity ignored). Longitudinal mode and shear horizontal mode are not affected by the piezoelectricity since the only nonzero piezoelectric constants of $\mathrm{Bi}_{12} \mathrm{GeO}_{20}$ are $e_{14}=e_{25}=e_{36}$. In this regards, Eq. (5) can be decoupled into the mixed polarization modes [i.e., longitudinal $(L)$ and shear horizontal $(\mathrm{SH})$ ] which are piezoelectric inactive and shear vertical (SV) modes with polarization of the displacement along the cylinder axis.

Based on Eq. (6), the electromechanical coupling coefficients for the surface mode and the pseudosurface mode are calculated as functions of $\theta$ and shown in Fig. 2(a). The pseudosurface branch has higher coupling coefficient than that of the surface branch with maxima 0.74 and $0.63 \%$ occur near [011] direction, respectively. The inset in Fig. 2(a) displays the angular dependence of dispersion curves of SAW, PSAW, and BAW at $k a / \pi=1$, dashed curves represent the shear vertical waves as piezoelectricity is ignored. For the case of constant reduced wave vector, the value of vertical axis of the inset is proportional to the phase velocity of the plane waves. In the figure, the SV wave remains almost constant, but the SH wave increases continuously with $\theta$ and maximized at $45^{\circ}$ from $\bar{\Gamma}-\bar{X}$ direction. For computation time consideration, we used 25 RLVs in all calculation conducted in this paper. The convergence test for the electromechanical coupling coefficient of the surface mode branch in this case shows that the satisfactory accuracy for the low-frequency part was attained.

Filling fraction is also a key parameter in controlling the electromechanical coupling coefficient. Figure 2(b) illustrates the variation of coupling coefficients of the surface mode and the pseudosurface mode at the $\bar{X}$ point as a function of the filling fraction, which are calculated along the surface mode and the pseudo-surface mode branches shown in the inset. In this propagating direction, the coupling coefficient is much smaller than that in other direction [see Fig. 2(a)]. The results in Fig. 2(b) show that the electromechanical coupling coefficient increases progressively with the increase of the filling fraction until a critical value and then decreases. The maximum $K^{2}$ values for the surface mode and the pseudo-surface mode are 0.047 and $0.163 \%$ at $f=0.549$ and $f=0.393$, respectively. The inset of Fig. 2(b) shows the band edges of the surface and pseudosurface acoustic wave frequency gap and its normalized width at the $\bar{X}$ point as a function of the filling fraction $f$. It shows that the maximum gap width appears at $f=0.183$ for short-circuit condition.
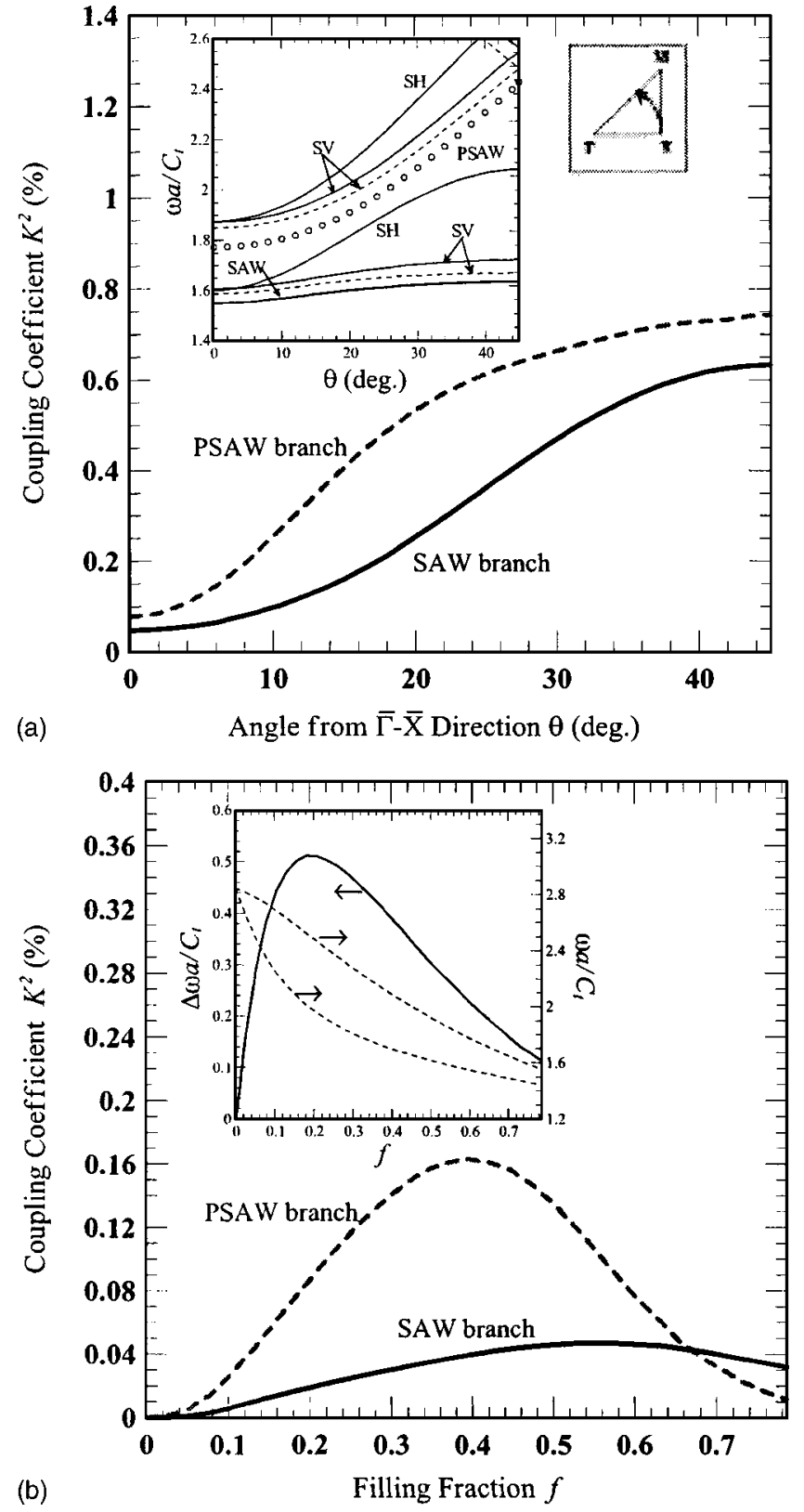

FIG. 2. Electromechanical coupling coefficients of the surface (solid line) and pseudosurface (dashed line) mode branches (a) as function of angle from $\bar{\Gamma}-\bar{X}$ direction at $k a / \pi=1$; (b) calculated along the frequency edges at the $\bar{X}$ point vs filling fraction $f$. The inset of (a) shows the angular dependence of the frequencies of the SAW, PSAW, and BAW modes. The inset of (b) shows the normalized gap width (bold solid line) and the band edges (dashed lines) of the gap in between the surface mode and the pseudosurface mode.

Note that when the filling fraction decreases to zero, the phase velocity of surface acoustic wave reduced to the SAW velocity of a single-crystal silica with no difference between the open-circuit and short-circuit conditions.

In piezoelectric phononic crystals, the periodic boundary condition causes the dispersive character for electromechanical coupling coefficient. Figure 3 shows the electromechanical coupling coefficient as function of frequency-lattice spacing product for the surface mode branch along the $\bar{\Gamma}-\bar{X}$ 


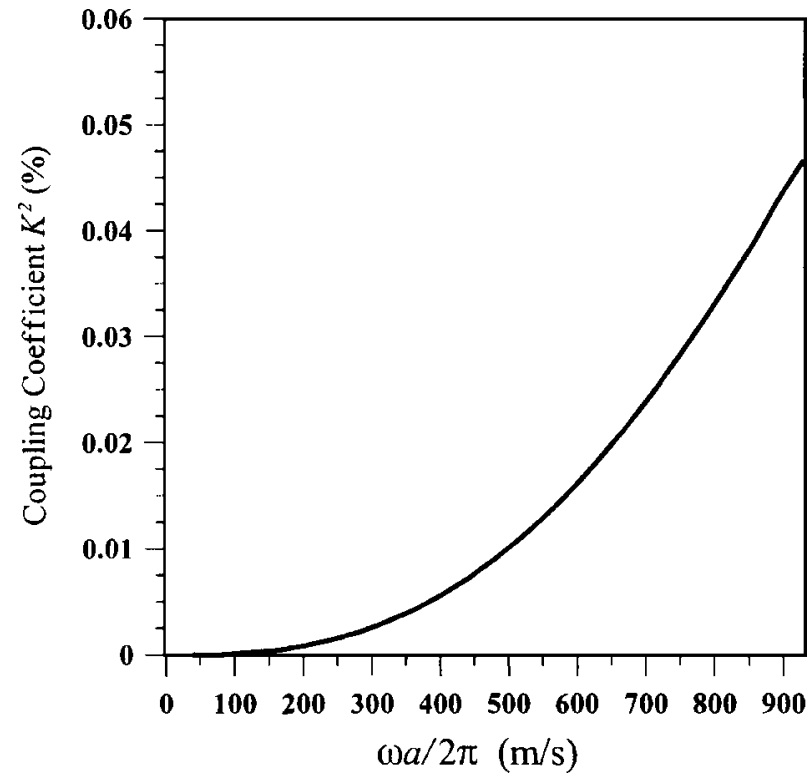

FIG. 3. The frequency dispersion of the electromechanical coupling coefficients for the first surface mode branch along the $\bar{\Gamma}-\bar{X}$ direction in the piezoelectric phononic crystal $\mathrm{Bi}_{12} \mathrm{GeO}_{20} / \mathrm{SiO}_{2}$.

direction in the piezoelectric phononic crystal $\mathrm{Bi}_{12} \mathrm{GeO}_{20} /$ $\mathrm{SiO}_{2}$. We note that the curve stop at the frequency of first surface mode branch corresponding to the symmetry point $\bar{X}$, that is, the frequency-lattice spacing product is equal to 929 $\mathrm{m} / \mathrm{s}$. The calculated results show that big change of coupling coefficients occur near the symmetry point $\bar{X}$ of the Brillouin zone. When the wavelength of surface acoustic wave compare with the lattice spacing, the effect of periodic boundary affect the variation of coupling coefficient apparently.

To conclude, we have calculated the phononic band structure of the bulk and surface waves in a two-dimensional periodic structure consisting of array of piezoelectric cylinders $\mathrm{Bi}_{12} \mathrm{GeO}_{20}$ in an isotropic background material $\mathrm{SiO}_{2}$. The explicit formulations of the bulk wave and the surface wave dispersion relations in such a structure were derived based on the plane wave expansion method. The band gaps and the electromechanical coupling coefficients for surface acoustic waves propagating in the phononic structure with piezoelectric filler material were calculated. In addition, the influences the filling fraction on the electromechanical coupling coefficients and band gap were evaluated. We found that the maximum band gap at the $\bar{X}$ point may appear at a filling ratio as small as 0.183 for the case of bismuth germanium oxide cylindrical filler in silica substrate. From the calculations, we note that there is no simple relation exists between the electromechanical coupling coefficient and filling ratio. To determine the optimal filling fraction of a particular piezoelectric phononic crystal, a detailed analysis is needed. Finally, we note that the results of this research offer extra design possibilities to control and enhance the performance of acoustic devices consisting of phononic structures.

The authors gratefully acknowledge the financial support from the National Science Council of Taiwan.

\section{APPENDIX}

The explicit expressions of matrix components in Eq. (5) and $\mathbf{H}$ are

$$
\begin{aligned}
& P_{\mathbf{G}, \mathbf{G}^{\prime}}^{(i, 4)}=P_{\mathbf{G}, \mathbf{G}^{\prime}}^{(4, i)}=-\left(G_{\alpha}+k_{\alpha}\right)\left(G_{\beta}^{\prime}+k_{\beta}\right) e_{\mathbf{G}-\mathbf{G}^{\prime}}^{\alpha \beta i}, \\
& P_{\mathbf{G}, \mathbf{G}^{\prime}}^{(4,4)}=\left(G_{\alpha}+k_{\alpha}\right)\left(G_{\beta}^{\prime}+k_{\beta}\right) \varepsilon_{\mathbf{G}-\mathbf{G}^{\prime}}^{\alpha \beta}, \\
& Q_{\mathbf{G}, \mathbf{G}^{\prime}}^{(i, 4)}=-\left[\left(G_{\alpha}+k_{\alpha}\right) e_{\mathbf{G}-\mathbf{G}^{\prime}}^{3 \alpha i}+\left(G_{\beta}^{\prime}+k_{\beta}\right) e_{\mathbf{G}-\mathbf{G}^{\prime}}^{\beta 3 i}\right], \\
& Q_{\mathbf{G}, \mathbf{G}^{\prime}}^{(4, i)}=-\left[\left(G_{\alpha}+k_{\alpha}\right) e_{\mathbf{G}-\mathbf{G}^{\prime}}^{\alpha i 3}+\left(G_{\beta}^{\prime}+k_{\beta}\right) e_{\mathbf{G}-\mathbf{G}^{\prime}}^{3 i \beta}\right], \\
& Q_{\mathbf{G}, \mathbf{G}}^{(4,4)}=-\left[\left(G_{\alpha}+k_{\alpha}\right) \varepsilon_{\mathbf{G}-\mathbf{G}^{\prime}}^{\alpha 3}+\left(G_{\beta}^{\prime}+k_{\beta}\right) \varepsilon_{\mathbf{G}-\mathbf{G}^{\prime}}^{3 \beta}\right], \\
& R_{\mathbf{G}, \mathbf{G}^{\prime}}^{(i, 4)}=R_{\mathbf{G}, \mathbf{G}^{\prime}}^{(4, i)}=-e_{\mathbf{G}-\mathbf{G}^{\prime}}^{33 i}, \quad R_{\mathbf{G}, \mathbf{G}^{\prime}}^{(4,4)}=\varepsilon_{\mathbf{G}-\mathbf{G}^{\prime}}^{33}, \\
& H_{i, \mathbf{G}}^{(m)}=\sum_{\mathbf{G}^{\prime}}\left[\begin{array}{c}
\left(G_{\alpha}^{\prime}+k_{\alpha}\right)\left(c_{\mathbf{G}-\mathbf{G}^{\prime}}^{i 3 j \alpha} a_{\mathbf{G}^{\prime}}^{j(m)}+e_{\mathbf{G}-\mathbf{G}^{\prime}}^{\alpha i 3} a_{\mathbf{G}^{\prime}}^{4(m)}\right) \\
+k_{z}^{(m)}\left(c_{\mathbf{G}-\mathbf{G}^{\prime}}^{i 3 j 3} a_{\mathbf{G}^{\prime}}^{j(m)}+e_{\mathbf{G}-\mathbf{G}^{\prime}}^{3 i 3} a_{\mathbf{G}^{\prime}}^{4(m)}\right)
\end{array}\right], \\
& H_{4, \mathbf{G}}^{(m)}=\sum_{\mathbf{G}^{\prime}}\left[\begin{array}{c}
\left(G_{\alpha}^{\prime}+k_{\alpha}\right)\left(e_{\mathbf{G}-\mathbf{G}^{\prime}}^{3 j \alpha} a_{\mathbf{G}^{\prime}}^{j(m)}-\varepsilon_{\mathbf{G}-\mathbf{G}^{\prime}}^{3 \alpha} a_{\mathbf{G}^{\prime}}^{4}\right) \\
+k_{z}^{(m)}\left(e_{\mathbf{G}-\mathbf{G}^{\prime}}^{3 j 3} a_{\mathbf{G}^{\prime}}^{j(m)}-\varepsilon_{\mathbf{G}-\mathbf{G}^{\prime}}^{33} a_{\mathbf{G}^{\prime}}^{4}\right)
\end{array}\right],
\end{aligned}
$$

for the open circuit and

$$
H_{4, \mathbf{G}}^{(m)}=a_{\mathbf{G}}^{4(m)}
$$

for the short circuit, where $i, j=1,2,3$, and $\alpha, \beta=1,2$.
*Email address: wutt@ ndt.iam.ntu.edu.tw

${ }^{1}$ M. S. Kushwaha, P. Halevi, L. Dobrzynski, and B. DjafariRouhani, Phys. Rev. Lett. 71, 2022 (1993).

${ }^{2}$ M. Wilm, A. Khelif, S. Ballandras, V. Laude, and B. DjafariRouhani, Phys. Rev. E 67, 065602(R) (2003).

${ }^{3}$ M. Kafesaki and E. N. Economou, Phys. Rev. B 60, 11993 (1999).

${ }^{4}$ I. E. Psarobas, N. Stefanou, and A. Modinos, Phys. Rev. B 62, 278 (2000)

${ }^{5}$ Z. Liu, C. T. Chan, P. Sheng, A. L. Goertzen, and J. H. Page,
Phys. Rev. B 62, 2446 (2000).

${ }^{6}$ J. Mei, Z. Liu, J. Shi, and D. Tian, Phys. Rev. B 67, 245107 (2003).

${ }^{7}$ D. García-Pablos, M. Sigalas, F. R. Montero de Espinosa, M. Torres, M. Kafesaki, and N. García, Phys. Rev. Lett. 84, 4349 (2000).

${ }^{8}$ Y. Tanaka and S. I. Tamura, Phys. Rev. B 58, 7958 (1998).

${ }^{9}$ Y. Tanaka and S. I. Tamura, Phys. Rev. B 60, 13294 (1999).

${ }^{10}$ E. V. Tartakovskaya, Phys. Rev. B 62, 11225 (2000).

${ }^{11}$ T.-T. Wu, Z.-G. Huang, and S. Lin, Phys. Rev. B 69, 094301 
(2004)

${ }^{12}$ M. Wilm, A. Khelif, S. Ballandras, and T. Pastureaud, J. Acoust. Soc. Am. 112, 943 (2002).

${ }^{13}$ J. H. Wilkinson, The Algebraic Eigenvalue Problem (Clarendon
Press, Oxford, 1965).

${ }^{14}$ K. A. Ingebrigtsen, J. Appl. Phys. 40, 2682 (1969).

${ }^{15}$ D. Royer and E. Dieulesaint, Elastic Waves in Solids I (SpringerVerlag, Berlin, 2000). 